\title{
Capacidade de Combinação de Características de Produção de Ovos de Linhagens de Matrizes de Frango de Corte, Usando Análise de Componentes Principais ${ }^{1}$
}

\section{Valéria Maria Nascimento Abreu ${ }^{2}$, Martinho de Almeida e Silva ${ }^{3}$, Cosme Damião $\mathrm{Cruz}^{4}$, Élsio Antônio Pereira de Figueiredo ${ }^{5}$, Paulo Giovanni de Abreu ${ }^{6}$}

\begin{abstract}
RESUMO - Este trabalho foi realizado com cruzamentos simples (dialelo completo) dentro de três linhagens fêmeas (PP, KK e VV). O delineamento experimental foi em blocos casualizados consistindo de nove cruzamentos, de três blocos de 81 aves. As características de produção de ovos, no total de doze, foram número de ovos da $24^{\mathrm{a}}-32^{\mathrm{a}}, 32^{\mathrm{a}}-40^{\mathrm{a}}, 40^{\mathrm{a}}-48^{\mathrm{a}}, 48^{\mathrm{a}}-56^{\mathrm{a}}, 56^{\mathrm{a}}-64^{\mathrm{a}}, 22^{\mathrm{a}}-66^{\mathrm{a}}, 32^{\mathrm{a}}-66^{\mathrm{a}}$, $40^{\mathrm{a}}-66^{\mathrm{a}}, 48^{\mathrm{a}}-66^{\mathrm{a}}, 24^{\mathrm{a}}-40^{\mathrm{a}}, 24^{\mathrm{a}}-48^{\mathrm{a}}$ e $24^{\mathrm{a}}-56^{\mathrm{a}}$ semana de vida das aves. Dois primeiros componentes principais explicaram mais de $98 \%$ da variação total das médias dos cruzamentos. No estudo da capacidade geral de combinação (CGC), com base nos escores dos dois primeiros componentes principais, foram selecionados pela CGC os progenitores PP e VV, para o componente principal1, e apenas o progenitor PP, para o componente principal 2.
\end{abstract}

Palavras-chave: componente principal, capacidade de combinação, produção de ovos, linhagens de frango de corte

\section{General Combining Ability of Egg Production Traits of Broiler Lines using Principal Components Analysis}

\begin{abstract}
This study was conducted using data from single crosses (diallel) withim three female lines (PP, KK and VV). A completely randomized block design, with 81 single crosses treatments, three replicales with 81 broilers was used. The twelve production traits were: egg number during the periods of $24^{\text {th }}-32^{\text {nd }}$, $32^{\text {nd }}-40^{\text {th }}, 40^{\text {th }}-48^{\text {th }}, 48^{\text {th }}-56^{\text {th }}, 56^{\text {th }}-64^{\text {th }}, 22^{\text {nd }}-66^{\text {th }}, 32^{\text {nd }}-66^{\text {th }}, 40^{\text {th }}-66^{\text {th }}, 48^{\text {th }}-66^{\text {th }}, 24^{\text {th }}-40^{\text {th }}, 24^{\text {th }}-$ $48^{\text {th }}$ and $24^{\text {th }}-56^{\text {th }}$ weeks of age. The first two principal components explained more than $98 \%$ of total variance of the crosses means. In the study of the general combining ability (GCA), based on the scores of the first two principal componentes, the parent lines PP and VV were selected by GCA, for principal component1, however for the principal component 2 the parent line selected was PP.
\end{abstract}

Key Words: principal component, combining ability, egg production traits, broiler lines

\section{Introdução}

A adoção dos componentes principais como critério para escolha de linhagens baseia-se no fato de ser uma estatística multivariada com propriedades interessantes para o melhoramento. Cada componente é uma combinação linear das características avaliadas e, portanto, considera, simultaneamente, $o$ conjunto de atributos de interesse. Outro fato, talvez o de maior interesse, é a propriedade de reter, nos primeiros componentes estimados, as maiores frações da variabilidade total disponível. Assim, como o sucesso do melhoramento reside na existência de variabilidade, acredita-se que a seleção baseada em um componente de alto poder discriminatório possa levar a resultado satisfatório.
Componentes principais são combinações lineares de variáveis aleatórias ou estatísticas, que possuem propriedades especiais, em termos de variâncias. Entre essas propriedades especiais, destaca-se o fato de que o primeiro componente principal possui a máxima variância, tendo o segundo a segunda maior variância e, assim, sucessivamente, de forma que o máximo de informação, em termos de variação total, esteja contido nos primeiros componentes. Além disso, os componentes principais são independentes entre si (ANDERSON, 1958).

Segundo BAKER et al. (1988), a análise dos componentes principais pode revelar relações não identificadas previamente, contribuindo para melhor interpretação dos dados. Além disso, muitas vezes, a mesma representa um método de explicação da es-

\footnotetext{
${ }^{1}$ Parte do trabalho de tese de D.S. do primeiro autor, financiada pela CAPES/UFV/EMBRAPA.

2 Pesquisador EMBRAPA/CNPSA, bolsista da CAPES.

3 Professor da Universidade Estadual do Norte Fluminense.

4 Professor da Universidade Federal de Viçosa.

5 Pesquisador EMBRAPA/CNPSA, bolsista do CNPq.

${ }^{6}$ Pesquisador EMBRAPA/CNPSA.
} 
trutura de covariância entre as várias características medidas em uma população animal.

Para CRUZ e REGAZZI (1994), a viabilidade de utilização dos componentes principais, em estudos sobre divergência genética, dependerá da possibilidade de resumir o conjunto de variáveis originais em poucos componentes, o que significará ter aproximação do comportamento dos indivíduos (progenitores), oriundos de um espaço $n$-dimensional ( $\mathrm{n}$ = número de caracteres estudados) em um espaço bi ou tridimensional. Quando esse requisito for satisfeito, a referida técnica proporcionará simplificação considerável nos cálculos estatísticos e na interpretação dos resultados com relação aos demais métodos alternativos, principalmente quando o número de progenitores avaliados for relativamente grande. Os indivíduos avaliados podem ser representados em um gráfico cartesiano bidimensional, sendo os eixos constituídos pelos escores dos dois primeiros componentes. Contudo, segundo CRUZ (1990), a avaliação da divergência em análise gráfica e o estabelecimento de grupos de similaridade com base na simples inspeção visual da dispersão são questionáveis e podem, às vezes, não ser tão claros na visualização do grau de similaridade entre os indivíduos estudados. Existem casos em que a análise não consegue resumir o complexo de informações das variáveis originais, ou seja, os dois ou três primeiros componentes principais contêm porcentagem da variância total insuficiente para avaliação segura da similaridade. Então, para se complementarem as informações da dispersão gráfica, são utilizadas técnicas de análise de agrupamento, que permitem o estabelecimento de grupos de maneira menos subjetiva que os exames visuais (CRUZ, 1990).

Na pesquisa agrícola a técnica teve sua exploração mais acentuada a partir da década de 70, devido à maior disponibilidade de recursos computacionais. Dessa forma, autores como MALUF e FERREIRA (1983), OLIVEIRA (1989) utilizaram a técnica de componentes principais em estudos com feijão e CARVALHO (1993) em algodão.

Os componentes principais são pouco utilizados na área animal e podem ser encontrados em estudos como de BAKER et al. (1988), examinando características de puberdade e de crescimento em touros e novilhas, e também ABEYWARDENA (1972), que recomendou a técnica como um método de estimação de repetibilidade em animais ou árvores. Estudos mais recentes foram feitos por FONSECA (1991), avaliando o crescimento das relações de alometria em linhagens especializadas na produção de frangos, e SAKAGUTI (1994), na avaliação de cruzamentos dialéticos em coelhos.

Objetivou-se com este trabalho estudar a capacidade de combinação entre linhagens de matrizes de frango de corte, por meio da técnica de componentes principais, utilizando dados de características de produção de ovos.

\section{Material e Métodos}

Os dados foram provenientes de experimento realizado no Setor de Melhoramento Genético de Aves (SMGA) do Centro Nacional de Pesquisa de Suínos e Aves (CNPSA), da Empresa Brasileira de Pesquisa Agropecuária (EMBRAPA), em Concórdia, Santa Catarina, no período de janeiro de 1994 a dezembro de 1995.

As aves deste experimento foram oriundas do cruzamento em dialelo completo dentro de três linhagens maternas (KK, PP e VV). Dessa forma, foram gerados nove cruzamentos das maternas, que foram alojadas em gaiolas.

\begin{tabular}{cc}
\hline \multirow{2}{*}{$\begin{array}{l}\text { Linhagem (linha materna) } \\
\text { Strain (maternal line) }\end{array}$} \\
\cline { 2 - 3 } \multicolumn{2}{c}{ Progenitor } \\
\cline { 2 - 3 } Macho & Fêmea \\
Male & Female \\
\hline $1-\mathrm{PP}$ & $1-\mathrm{PP}$ \\
$2-\mathrm{KK}$ & $2-\mathrm{KK}$ \\
$3-\mathrm{VV}$ & $3-\mathrm{VV}$ \\
\hline
\end{tabular}

$\mathrm{O}$ delineamento experimental foi em blocos casualizados, consistindo de nove cruzamentos, três blocos de 81 aves.

As 12 características de produção de ovos estudadas foram número de ovos da $24^{\mathrm{a}}-32^{\mathrm{a}}(\mathrm{P} 1) ; 32^{\mathrm{a}}-40^{\mathrm{a}}$ $(\mathrm{P} 2) ; 40^{\mathrm{a}}-48^{\mathrm{a}}(\mathrm{P} 3) ; 48^{\mathrm{a}}-56^{\mathrm{a}}(\mathrm{P} 4) ; 56^{\mathrm{a}}-64^{\mathrm{a}}(\mathrm{P} 5)$; $22^{\mathrm{a}}-66^{\mathrm{a}}(\mathrm{P} 6) ; 32^{\mathrm{a}}-66^{\mathrm{a}}(\mathrm{P} 7) ; 40^{\mathrm{a}}-66^{\mathrm{a}}(\mathrm{P} 8) ; 48^{\mathrm{a}}-66^{\mathrm{a}}$ (P9); 24 $4^{\mathrm{a}}-40^{\mathrm{a}}(\mathrm{P} 10) ; 24^{\mathrm{a}}-48^{\mathrm{a}}(\mathrm{P} 11)$ e $24^{\mathrm{a}}-56^{\mathrm{a}}(\mathrm{P} 12)$ semana de vida das aves. Estas características foram obtidas por meio de anotações diárias do número de ovos.

As análises foram realizadas utilizando-se o programa GENES (CRUZ, 1997).

A técnica de componentes principais consiste em transformar o conjunto de $\mathrm{n}$ variáveis padronizadas $x_{i 1}, x_{i 2}, \ldots, x_{i n}$ em um novo conjunto de $Y_{i 1}, Y_{i 2}, \ldots, Y_{i n}, \mathrm{em}$ que os $Y_{i^{\prime} s}$ são funções lineares do $x_{i \text { 's }}$ e independentes entre si.

As seguintes propriedades são verificadas:

a) $\mathrm{Se} \mathrm{Y}_{\mathrm{ij}}$ é um componente principal, então:

$$
Y_{i j}=a_{1} x_{i 1}+a_{2} x_{i 2}+\ldots+a_{n} x_{i n}
$$

b) Se $Y_{\mathrm{ij}}$ 'é outro componente principal, então:

$$
Y_{i j}{ }^{\prime}=b_{1} x_{i 1}+b_{2} x_{i 2}+\ldots+b_{n} x_{i n}
$$




$$
\sum a_{j}^{2}=\sum b_{j}^{2}=1
$$

$\sum \mathrm{a}_{\mathrm{j}} \mathrm{b}_{\mathrm{j}}=0$, ou seja, os componentes são nãocorrelacionados.

c) Entre todos os componentes, $\mathrm{Y}_{\mathrm{i} 1}$ apresenta a maior variância; $\mathrm{Y}_{\mathrm{i} 2}$, a segunda maior e assim sucessivamente.

CRUZ e REGAZZI (1994) mostram que os componentes principais podem ser obtidos pelo sistema:

$$
\operatorname{det}=\left(R-\lambda_{1} I\right) a=0
$$

em que

$\mathrm{R}=$ matriz de correlação entre as médias estimadas dos cruzamentos;

$\lambda_{1}=$ raízes características (ou autovalores) de $\mathrm{R}$; e

$\mathrm{a}=$ vetor característico (ou autovetor) associado aos autovalores.

Assim, os autovalores de $\mathrm{R}$ correspondem às variâncias de cada componente e os autovetores normalizados correspondem aos coeficientes de ponderação dos caracteres padronizados.

Após a determinação do número de componentes principais, que envolveram um mínimo de $80 \%$ da variação disponível, foram estimados os escores para cada componente principal. A importância relativa de um componente, que é avaliada pela percentagem da variância total que esse explica, foi calculada por:

Importância de $Y_{j}=\frac{\mathrm{V}\left(\mathrm{Y}_{\mathrm{j}}\right)}{\sum_{\mathrm{j}=1}^{\mathrm{n}} \mathrm{V}\left(\mathrm{Y}_{\mathrm{j}}\right)}=\frac{\lambda_{\mathrm{j}}}{\sum_{\mathrm{j}=1}^{\mathrm{n}} \lambda_{\mathrm{j}}}=\frac{\lambda_{\mathrm{j}}}{\operatorname{Tr}(\mathrm{R})}$

Em seguida, foi realizada a análise (univariada) dialélica dos escores dos componentes principais que envolveram, no mínimo, $80 \%$ da variação total e, também, foram determinadas as estimativas do efeito da capacidade geral de combinação $\left(\hat{\mathrm{g}}_{\mathrm{i}}\right)$ e da capacidade especifica de combinação $\left(\hat{\mathrm{s}}_{\mathrm{ii}}\right.$ e $\left.\hat{\mathrm{s}}_{\mathrm{ij}}\right)$, recíproco $\left(\hat{\mathrm{r}}_{\mathrm{ij}}\right)$ e média (F1 e recíproco) dos dois primeiros componentes principais.

\section{Resultados e Discussão}

Com apenas dois componentes principais, foi possível reunir mais de $98 \%$ da variação total disponível entre as médias de cruzamentos, em que o primeiro componente foi responsável por cerca de $66 \%$ e o segundo, aproximadamente, por $32 \%$ dessa variação, como mostrado na Tabela 1.

Na Tabela 2, são apresentados os coeficientes de ponderação de cada característica e suas correlações com os dois primeiros componentes principais.

Observa-se que o primeiro componente está mais correlacionado com o período total de produção (P6), que é a característica de grande importância no estudo de matrizes para corte, mas, também, apresenta alta correlação com várias outras características. Esse componente principal, então, é o componente denominado de produção total. O segundo componente está mais correlacionado com P1 (24-32 semanas) e P10 (24-40 semanas), ou seja, períodos em que as aves iniciam e chegam ao pico de postura. Por essa razão, esse componente, neste estudo, é denominado de componente de pico de postura.

Pela Tabela 3, observa-se que os maiores escores, para o primeiro componente principal, foram

Tabela 1 - Autovalores, explicação e percentagem acumulada dos componentes principais

Table 1 - Eigenvalues, percentage and accumulated percentage of total variance accounted by principal components

\begin{tabular}{lccc}
\hline $\begin{array}{l}\text { Componente } \\
\text { principal } \\
\begin{array}{l}\text { Principal } \\
\text { component }\end{array}\end{array}$ & $\begin{array}{c}\text { Autovalores } \\
\text { Eigenvalues }\end{array}$ & $\begin{array}{c}\text { Explicação(\%) } \\
\text { Percentage oftotal } \\
\text { variance }\end{array}$ & $\begin{array}{c}\text { Porcentagem acumulada(\%) } \\
\text { Accumulated } \\
\text { percentage }\end{array}$ \\
\hline CP1 & 7,985514 & 66,54 & 66,54 \\
CP2 & 3,830199 & 31,92 & 98,46 \\
CP3 & 0,138591 & 1,15 & 99,61 \\
CP4 & 0,040215 & 0,33 & 99,95 \\
CP5 & 0,005259 & 0,04 & 99,99 \\
CP6 & 0,000143 & 0,01 & 100,00 \\
CP7 & 0,000110 & 0,00 & 100,00 \\
CP8 & 0,000077 & 0,00 & 100,00 \\
CP9 & 0,000058 & 0,00 & 100,00 \\
CP10 & 0,000002 & 0,00 & 100,00 \\
CP11 & 0,000069 & 0,00 & 100,00 \\
CP12 & 0,000106 & 0,00 & 100,00 \\
\hline
\end{tabular}


ABREU et al.

Tabela 2 - Coeficientes de ponderação das características $(C)$ e seus coeficientes de correlações (r) com os dois primeiros componentes principais (produção total e pico de postura)

Table 2 - Weighting coefficients of the traits and their correlation with the two principal components (egg production and egg production peak

\begin{tabular}{|c|c|c|c|c|}
\hline \multirow[t]{2}{*}{$\begin{array}{l}\text { Característica } \\
\text { Trait }\end{array}$} & \multicolumn{2}{|c|}{$\begin{array}{l}\text { Produção total } \\
\text { Total production }\end{array}$} & \multicolumn{2}{|c|}{$\begin{array}{c}\text { Pico de postura } \\
\text { Egg production peak }\end{array}$} \\
\hline & $\mathrm{C}$ & $\mathbf{r}$ & C & $\mathbf{r}$ \\
\hline $\mathrm{P} 1$ & 0,0744 & 0,2099 & 0,4970 & 0,9729 \\
\hline P2 & 0,3228 & 0,9122 & 0,1788 & 0,3501 \\
\hline P3 & 0,3238 & 0,9149 & $-0,1706$ & $-0,3334$ \\
\hline P4 & 0,3173 & 8,8966 & $-0,2100$ & $-0,4112$ \\
\hline P5 & 0,3133 & 0,8855 & $-0,2208$ & $-0,4321$ \\
\hline P6 & 0,3397 & 0,9600 & 0,1393 & 0,2728 \\
\hline P7 & 0,3381 & 0,9559 & $-0,1497$ & $-0,2931$ \\
\hline P8 & 0,3205 & 0,9059 & $-0,2155$ & $-0,4221$ \\
\hline P9 & 0,3151 & 0,8906 & $-0,2256$ & $-0,4415$ \\
\hline P10 & 0,1455 & 0,4113 & 0,4653 & 0,9107 \\
\hline P11 & 0,2203 & 0,6228 & 0,3997 & 0,7821 \\
\hline P12 & 0,2913 & 0,8234 & 0,2887 & 0,5649 \\
\hline
\end{tabular}

obtidos pelos cruzamentos 13 e 33. Já para o segundo componente, os maiores escores foram obtidos pelos cruzamentos 11 e 31 .

Em razão de os resultados evidenciarem que os dois primeiros componentes envolvem mais de $98 \%$ da variação total disponível, obtém-se uma análise satisfatória da divergência genética, por meio da dispersão dos escores em gráficos cujos eixos são os referidos componentes.

A dispersão desses escores em eixos cartesianos é apresentada na Figura 1.

O gráfico de dispersão dos cruzamentos mostra que existe divergência genética entre os mesmos. Basicamente foram formados três grupos, o primeiro com os cruzamentos 13,12 e 31 , o segundo com os cruzamentos 33 e 32 e o terceiro 21,22 e 23 com o cruzamento 11 isolado.

$\mathrm{Na}$ Tabela 4, são apresentadas estimativas dos efeitos da capacidade geral de combinação $\left(\hat{\mathrm{g}}_{\mathrm{i}}\right)$, capacidade específica de combinação $\left(\hat{\mathrm{s}}_{\mathrm{ii}}\right.$ e $\left.\hat{\mathrm{s}}_{\mathrm{ij}}\right)$ e recíproco $\left(\hat{\mathrm{r}}_{\mathrm{ij}}\right)$ e a média dos cruzamentos $(\mathrm{F} 1 \mathrm{e}$ recíproco), obtidos a partir dos escores dos dois primeiros componentes principais.

Pelo componente principal 1(CP1) seriam selecionados pela $\mathrm{CGG}$ os progenitores 1 e 3 , ao passo que, pelo componente principal 2 (CP2), apenas o progenitor 1 seria selecionado.

Os híbridos superiores e progenitores selecionados para serem usados como macho ou fêmea, nos cruzamentos, de acordo com os dois primeiros componentes principais, são apresentados a seguir:
Tabela 3 - Escores dos cruzamentos para os dois primeiros componentes principais

Table 3 - Scores of crosses for the two first principal components

\begin{tabular}{|c|c|c|}
\hline \multirow{3}{*}{$\begin{array}{l}\text { Cruzamento } \\
\text { Cross }\end{array}$} & \multicolumn{2}{|c|}{$\begin{array}{l}\text { Escore } \\
\text { Score }\end{array}$} \\
\hline & Produção total & Pico de postura \\
\hline & Total production & Egg production peak \\
\hline$\overline{11}$ & 73,0729 & 15,1507 \\
\hline 12 & 74,7975 & 11,5841 \\
\hline 13 & 77,2960 & 12,9811 \\
\hline 21 & 69,2364 & 11,7621 \\
\hline 22 & 70,5162 & 10,2295 \\
\hline 23 & 72,0732 & 11,2791 \\
\hline 31 & 75,0281 & 13,7852 \\
\hline 32 & 74,8433 & 9,1276 \\
\hline 33 & 77,3988 & 9,7546 \\
\hline
\end{tabular}

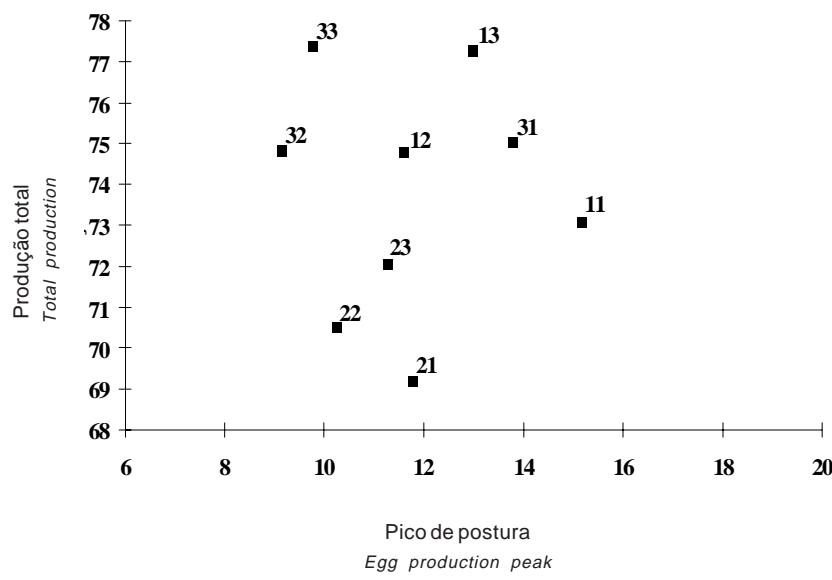

Figura 1 - Gráfico de dispersão dos cruzamentos em relação ao seus escores, nos dois componentes principais (produção total e pico de postura).

Figure 1 - Distribution of the crosses based in the scores of the two first principal component (total production and egg production peak). 
Rev. bras. zootec.

Tabela 4 - Estimativas do efeito da capacidade geral de combinação $\left(\hat{\mathrm{g}}_{\mathrm{i}}\right)$, capacidade especifica de combinação $\left(\hat{\mathrm{S}}_{\mathrm{ii}}\right.$ e $\left.\hat{\mathrm{S}}_{\mathrm{ij}}\right)$, recíproco $\left(\hat{\mathrm{r}}_{\mathrm{ij}}\right)$ e média ( $\mathrm{F} 1$ e recíproco) dos dois primeiros componentes principais

Table 4 - General $\left(\hat{\mathrm{g}}_{\mathrm{i}}\right)$ and specific combining ability $\left(\hat{\mathrm{S}}_{\mathrm{ii}}\right.$ e $\left.\hat{\mathrm{S}}_{\mathrm{ij}}\right)$ and reciprocal effects ( $\left.\hat{\mathrm{r}}_{\mathrm{ij}}\right)$ and crosses mean (F1 and reciprocal) estimates for the two principal components

\begin{tabular}{|c|c|c|c|c|c|c|c|c|}
\hline \multirow{3}{*}{$\begin{array}{l}\text { Cruzamento } \\
\text { Cross }\end{array}$} & \multicolumn{4}{|c|}{ CP1 } & \multicolumn{4}{|c|}{$\mathrm{CP} 2$} \\
\hline & \multicolumn{2}{|c|}{$\begin{array}{l}\text { Média } \\
\text { Mean }\end{array}$} & \multicolumn{2}{|r|}{$\begin{array}{l}\text { Efeito } \\
\text { Effect }\end{array}$} & \multicolumn{2}{|r|}{$\begin{array}{l}\text { Média } \\
\text { Mean }\end{array}$} & \multicolumn{2}{|c|}{$\begin{array}{l}\text { Efeito } \\
\text { Effect }\end{array}$} \\
\hline & F1 & $\operatorname{Rec}$ & $\hat{\mathrm{S}}_{\mathrm{ij}}$ & $\hat{\mathrm{r}}_{\mathrm{ij}}$ & F1 & Rec & $\hat{\mathrm{S}}_{\mathrm{ij}}$ & $\hat{\mathrm{r}}_{\mathrm{ij}}$ \\
\hline$\overline{12}$ & 270,101 & 249,321 & $-0,042$ & 10,390 & 67,712 & 66,493 & $-2,707$ & 0,609 \\
\hline 13 & 279,683 & 271,815 & 2,045 & 3,934 & 73,990 & 76,567 & 2,525 & $-1,288$ \\
\hline \multirow[t]{2}{*}{23} & 259,252 & 269,842 & $-1,507$ & $-5,295$ & 65,497 & 58,252 & 0,486 & 3,622 \\
\hline & \multicolumn{2}{|c|}{$\begin{array}{l}\text { Média } \\
\text { Mean }\end{array}$} & $\hat{\mathrm{g}}_{\mathrm{i}}$ & $\hat{\mathrm{S}}_{\mathrm{ii}}$ & \multicolumn{2}{|r|}{$\begin{array}{l}\text { Média } \\
\text { Mean }\end{array}$} & $\hat{\mathrm{g}}_{\mathrm{i}}$ & $\hat{\mathrm{S}}_{\mathrm{ii}}$ \\
\hline 11 & \multicolumn{2}{|c|}{265,401} & 0,450 & $-2,002$ & \multicolumn{2}{|r|}{81,357} & 6,595 & 0,182 \\
\hline 22 & \multicolumn{2}{|c|}{253,653} & $-7,200$ & 1,549 & \multicolumn{2}{|r|}{60,666} & $-4,770$ & 2,221 \\
\hline 33 & \multicolumn{2}{|c|}{279,467} & 6,750 & $-0,538$ & & 61,323 & $-1,825$ & $-3,010$ \\
\hline
\end{tabular}

\begin{tabular}{|c|c|c|c|}
\hline \multirow[b]{2}{*}{$\begin{array}{l}\text { Carac. } \\
\text { Charac. }\end{array}$} & \multirow[b]{2}{*}{$\begin{array}{c}\text { Híbrido superior } \\
\text { Superior hibrid }\end{array}$} & \multicolumn{2}{|c|}{$\begin{array}{c}\text { Progenitor a ser } \\
\text { utilizado como } \\
\text { Progenitor to be used as }\end{array}$} \\
\hline & & $\begin{array}{c}\text { Macho } \\
\text { Male }\end{array}$ & $\begin{array}{c}\text { Fêmea } \\
\text { Female }\end{array}$ \\
\hline$\overline{\mathrm{CP} 1}$ & 13 & 1 & 3 \\
\hline CP2 & 31 & 3 & 1 \\
\hline
\end{tabular}

\section{Conclusões}

Com apenas dois componentes principais, foi possível reunir mais de $98 \%$ da variação total disponível entre as médias de cruzamentos.

Foram selecionados pela CGC os progenitores PP e VV, para o componente principal 1, e apenas o progenitor PP, para o componente principal 2.

A análise por componentes principais mostrou-se eficiente neste estudo, acreditando-se que a seleção com base no componente de alto valor discriminatório pode levar a resultado satisfatório.

\section{Referências Bibliográficas}

ABEYWARDENA, V. 1972. An application of principal component anlysis in genetics. J. Genet., 61:27-51.

ANDERSON, T.W. 1958. An introduction to multivariate statistical analysis. New York, John Wiley \& Sons, 374p.

BAKER, J.F., STEWART, T.S., LONG, C.R. et al. 1988. Multiple regression and principal componentes analysis of puberty and growth in cattle. J. Anim. Sci., 66(9):2147-2158.

CARVALHO, L.P. Divergência genética e análise dialélica em Gossypium hirsutum var.latifolium hutch. Viçosa, MG: UFV,
1993. 203p. Tese (Doutorado em Melhoramento Genético) Universidade Federal de Viçosa, 1993.

CRUZ, C.D., REGAZZI, A.J. 1994. Modelos biométricos aplicados ao melhoramento genético. 1.ed., Viçosa: UFV. 390p.

CRUZ, C. D. Aplicação de algumas técnicas multivariadas no melhoramento de plantas. Piracicaba, SP: ESALQ, 1990. 188p. Tese (Doutorado em Melhoramento Genético) - Escola Superior de Agricultura "Luiz de Queiroz", 1990.

CRUZ, C.D. 1997. Programa GENES-Aplicativo computacional em genética e estatística. Viçosa, MG, UFV, 442p.

FONSECA, C.G. Análise de crescimento e relações de alometria em aves de corte. Ribeirão Preto, SP: USP, 1991.94p. Tese (Doutorado em Melhoramento Genético) - Faculdade de Medicina de Ribeirão Preto, 1991.

MALUF, W.R., FERREIRA, P.E. 1983. Análise multivariada da divergência genética em feijão vagem (Phaseolus vulgaris $L$.). Hort. Bras., 1:31-34.

OLIVEIRA, E. J.Análise multivariada no estudo da divergência genética entre cultivares de feijão. Viçosa, MG: UFV, 1989. 91p. Dissertação (Mestrado em Melhoramento Genético) Universidade Federal de Viçosa, 1989.

SAKAGUTI, E. S. Utilização de técnicas de análise multivariada na avaliação de cruzamentos dialéticos em coelhos. Viçosa, MG: UFV, 1994. 170p. Dissertação (Mestrado em Melhoramento Genético) - Universidade Federal de Viçosa, 1994.
Recebido em: 24/09/98

Aceito em: 09/03/99 